\title{
Successful device retrieval using simple balloon method during cardiac procedures
}

\author{
SHIVANAND PATIL*, NATRAJ SETTY, RANGARAJ RAMALINGAM, \\ JAYASHEELAN MAMBALLY, CHOLENAHALLY NANJAPPA MANJUNATH
}

\begin{abstract}
Department of Cardiology, Sri Jayadeva Institute of Cardiovascular Sciences and Research, Bangalore, India
*Corresponding author: Dr. Shivanand Patil, MBBS, MD, DM, FESC, FSCAI, FICC, Associate Professor; Department of Cardiology, Sri Jayadeva Institute of Cardiovascular Sciences and Research, Jayanagar 9th Block, Bannerghatta Road, Bangalore 560 069, Karnataka, India; Phone: +91 974016 6122; Fax: +91 802653 4477; E-mail: drssspatil@rediffmail.com
\end{abstract}

(Received: July 28, 2017; Revised manuscript received: March 20, 2018; Second revised manuscript received: April 26, 2018; Third revised manuscript received: June 8, 2018; Accepted: June 12, 2018)

\begin{abstract}
Objectives: Although rare, incidents of broken/dislodged fragment of various angioplasty hardwares, including catheters, guidewires, angioplasty balloons, and stents, are being increasingly reported in recent years. Since these broken fragments may cause life-threatening consequences for a patient, it is vital for an interventional cardiologist to be acquainted with different retrieval techniques. Here, we are reporting our observations of several incidents of device dislodgement/fracture during cardiac interventions and their retrieval using simple balloon method. Methods: We present a study of eight patients in whom we attempted to retrieve dislodged/fractured cath-lab hardwares during cardiac interventions, using simple balloon method. These cases include two cases of balloon, three cases of stent, and three cases of guidewire dislodgement/fracture. Results: Fractured/dislodged cath-lab hardwares were successfully retrieved using a simple balloon method in six out of eight cases $(75 \%)$, without any need of other retrieval hardwares. We observed no major complications in any patient. Conclusions: The balloon-assisted retrieval method is a simple, safe, and cost-effective way to avoid complications of endothelial injury, myocardial infarction, emergency coronary artery bypass graft, and sudden cardiac death. This study, particularly the context of retrieval technique used in each case, will offer valuable information to fellow interventional cardiologists.
\end{abstract}

Keywords: balloon mitral valvotomy, detached post-dilation balloon, percutaneous retrieval, broken stent shaft, uncoiled guidewire

\section{Introduction}

Coronary angiography, percutaneous transluminal coronary angioplasty (PTCA), and other percutaneous cardiac procedures are relatively safe as compared to surgical treatment [1]. However, increasing application of these interventions in managing complex lesions is plagued by unpleasant events of broken/dislodged fragments of various hardware, including catheters, guidewires, angioplasty balloons, and stents [2]. Such incidents are reported in about $0.1 \%-0.8 \%$ cases of coronary interventions [1]. Removal of the broken fragment becomes mandatory as they can lead to potentially life-threatening consequences of acute occlusion, myocardial infarction, arrhythmias, perforation, and thromboembolism [2]. Since such incidents can cause panic in the catheterization laboratory, it is vital for an interventional cardiologist to be acquainted with retrieval techniques $[2,3]$. Recently, we observed several incidents of device dislodgement/ rupture at our cath-lab. We are reporting our observations and retrieval techniques for such patients in this study. It is important to note that all these cases were managed with simple balloon method.

\section{Methods}

Study design and patient population

This was a hospital-based, retrospective study conducted at the Department of Cardiology, Sri Jayadeva Institute of Cardiovascular Sciences and Research, Bangalore, India between January 2011 and December 2015. Study population comprised patients who fulfilled all of the

This is an open-access article distributed under the terms of the Creative Commons Attribution-NonCommercial 4.0 International License, which permits unrestricted use, distribution, and reproduction in any medium for non-commercial purposes, provided the original author and source are credited, a link to the CC License is provided, and changes - if any - are indicated. 
following inclusion criteria: (1) patients undergoing percutaneous cardiac intervention, (2) incidents of broken/ dislodged fragment of any hardware during the percutaneous cardiac intervention, (3) attempt to retrieve the broken/dislodged fragment of any hardware using simple balloon method. The authors of this manuscript have certified that they comply with the principles of ethical publishing in Interventional Medicine \& Applied Science. All patients provided informed consent for the procedure and subsequent data collection and analysis for the research purposes.

\section{Data collection}

All data related to demographic information, comorbidities, procedure and cath-lab equipment details, information about complications of broken/dislodged fragments of a cath-lab equipment in the coronary artery, procedure details of device retrieval, and data on post-procedural stay for each patient examined in the study were collected from the hospital records, comprising angiography angioplasty reports, discharge summary, and procedure CDs. For any additional details, the operators were contacted for individual cases.

\section{Simple balloon method of device retrieval}

In general, the simple balloon method for retrieval involves introduction of a balloon over the guidewire to a point slightly beyond the broken/dislodged fragments of cath-lab hardware. Then, the balloon is inflated at appropriate pressure to trap the broken fragments of cathlab hardware between the inner wall of the guiding catheter and the outer surface of the balloon. Subsequently, correct pressure is maintained to assist en masse pulling out of entire assembly from the vascular system, leading to removal/retrieval of broken fragments of cathlab hardware. The representative figure for the simple balloon method of percutaneous device retrieval is illustrated in Fig. 1.

\section{Study endpoints}

The effectiveness endpoint of the simple balloon method of percutaneous device retrieval was defined as successful removal/retrieval of broken fragments of cath-lab hardware from the vascular system. The safety endpoint of the simple balloon method of percutaneous device retrieval was defined as procedural success without occurrence of any clinically significant events like dissection/perforation of coronary arteries.

\section{Statistical analysis}

Continuous variables are presented as mean \pm standard deviation, whereas categorical variables are expressed as percentages. All data were processed using the Statistical Package for Social Sciences software, version 15 (SPSS, Chicago, IL, USA).
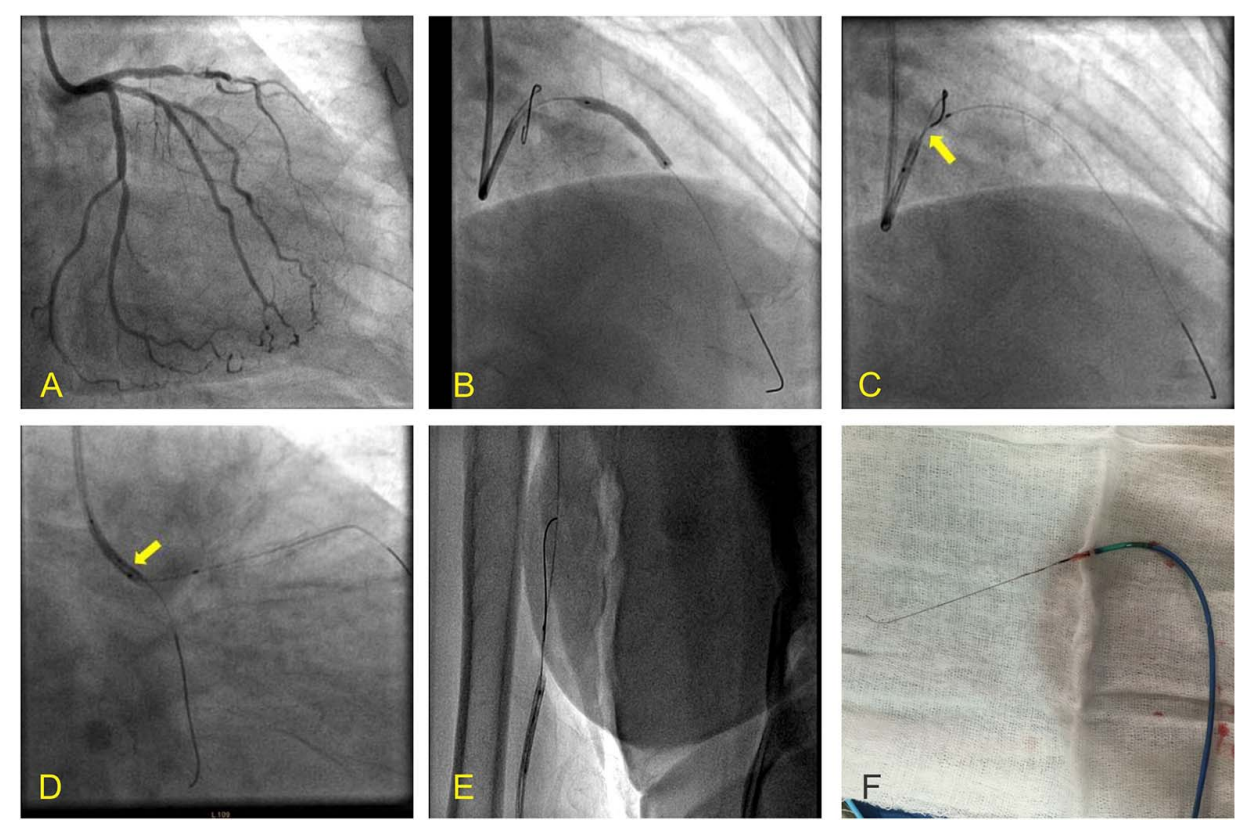

Fig. 1. (A) Significant occlusion in mid left anterior descending artery (LAD); (B) A $3 \times 23 \mathrm{~mm}$ drug-eluting stent deployed in LAD lesion; (C) Detached balloon remained in left main coronary artery after post-dilatation; (D) Detached balloon was trapped between outer surface of the non-compliant balloon balloon and inner surface of the catheter; (E) Whole assembly was removed en masse through the radial sheath; (F) Retrieved balloon after its removal from the vascular system 


\section{Patil et al.}

\section{Results}

During the study period, we encountered eight patients in whom dislodged/fractured cath-lab hardware retrieval was attempted using simple balloon method. The individual details of each of these cases are given in Table I. In brief, the mean age of these patients was $50.4 \pm 12.4$ years, and three $(37.5 \%)$ of them were females. Simple balloon method was attempted to retrieve detached PTCA balloons in two cases, broken/uncoiled guidewires in three cases, and broken/dislodged stents in three cases. The average length and diameter of the balloons used for the device retrieval were $10.4 \pm 1.9$ and $2.1 \pm$ $0.5 \mathrm{~mm}$, respectively. The mean inflation pressure used for the simple balloon retrieval method for cath-lab devices was $13.1 \pm 2.0 \mathrm{~atm}$. Of these patients, the device retrieval using simple balloon method was successful in six cases. Hence, the successful retrieval rate was $75 \%$ and the ratio of successful retrieval was 3:1. Of two cases where the attempts to retrieve the broken dislodged cath-lab devices with simple balloon method were unsuccessful, the broken guidewire was retrieved successfully using another method (i.e., paired guidewires knotted together) in one case, while a non-inflated dislodged stent got slipped out of the coronary system and was lost in a branch of radial artery in forearm in the other case, with no further attempts made to retrieve the dislodged stent, considering it was out of coronary circulation.

\section{Discussion}

The retrieval of broken/fractured fragments of cath-lab equipment is infrequently reported in literature [2-4]. There has been no evidence-based approach on which technique should be used for the immediate removal of the entrapped devices during percutaneous cardiac intervention. Furthermore, vigorous efforts for percutaneous retrieval can also create further complications in the already diseased vessel. Moreover, some cardiologists with limited interventional experiences may prefer urgent cardiothoracic surgery. However, we believe that cardiothoracic surgery should be considered as the last resort and attempts should be made for percutaneous retrieval $[3,5]$. Since there is no single technique, which will succeed in all situations, interventionists should be familiar with different percutaneous methods for the retrieval $[6,7]$. The commonly used percutaneous retrieval techniques include the use of baskets, snare loop, goose-neck snare, indigenously made loop snare, paired guidewires knotted together, double or triple wire technique, tornus catheter, and biopsy forceps $[5,6]$. It is noteworthy that these methods are associated with needs for additional hardware, increased procedural cost, and concern of thrombotic events or injury to coronary arteries leading to repeated revascularization [3]. Hence, we usually do

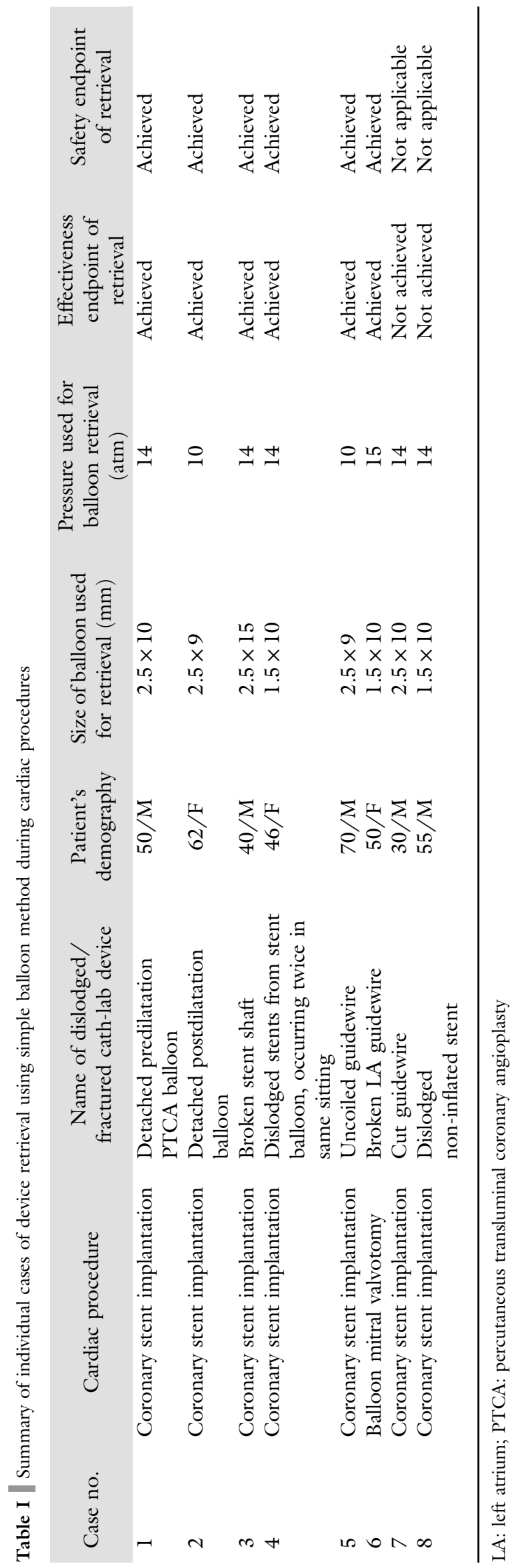


not opt for them. In addition, snares can be effective only when the lost device is in proximal most parts of coronary arteries. On the contrary, the simple balloon method of device retrieval is quite easy, less cumbersome, and safer than other described techniques with the use of an additional angioplasty wire and a balloon catheter.

Earlier, Chang et al. [8] had described two cases of retrieval of ruptured coronary balloon entrapment using simple balloon method. Brilakis et al. [9] reported the incidents of stent loss in 38 of 11,773 percutaneous coronary intervention (PCI) procedures $(0.32 \%$; 95\% confidence interval: $0.23 \%-0.44 \%)$. These incidents were found to be more common in lesions with calcification and/or significant proximal angulation. Lost stents were successfully retrieved in $86 \%$ cases using a variety of retrieval techniques. Another systemic review and metaanalysis by Alomar et al. [10] reported that stent loss had occurred in 919 of 71,655 PCIs (1.3\%; 95\% confidence interval: $0.8 \%-2.8 \%)$ and stents were successfully retrieved in majority of these cases. In line with this, we were able to retrieve broken/dislodged cath-lab hardwares during cardiac procedures using simple balloon method in $75 \%$ cases in this study. We believe that this study would provide valuable information on technical challenges and use of simple balloon method of device retrieval.

The major learning points from this study can be as follows: (1) interventionists should not only be aware of the possibility of device entrapment and device loss during complex interventions, but also should be familiar with different percutaneous methods for the retrieval; (2) the cases highlight the importance of adequate lesion assessment and preparation; (3) a little too much aggression on the part of the operator should be avoided while introducing or withdrawing various cath-lab devices; (4) although hardware reuse is difficult to avoid situation in developing countries where cost factor is an issue, it should be avoided as and when possible during interventions; (5) once a broken fragment is detected, all attempts should be made to retrieve it quickly and also to prevent it from further slipping at the distal ends of the vessel; (6) retrieval systems should be available in all interventional catheterization laboratories; (7) during retrieval attempts, monitoring of activated clotting time and maintaining it at least $50 \mathrm{~s}$ higher than the usual time are essential to prevent thrombus formation around the entrapped devices; and (8) vigorous efforts for percutaneous retrieval can also create further risk of endothelial injury, dissection, and thrombosis to the already diseased vessel endothelium. Hence, it should also be understood that such techniques are technically challenging and sometimes even unsuccessful.

Overall, the incidents of dislodgement of cath-lab equipment during the procedure may occur due to patient-related factors (i.e., calcified or tortuous vessel and complex lesion), operator-related factors (i.e., inadequate predilation or excessive force), or device-manufacturing defects [5]. In this study, the probable reasons for dislodgement/fracture of these devices could be significant calcification in the lesion, aggressive pushing, reuse of cathlab devices, stent overlapping to underexpanded stent, and manufacturing flaws. Using simple balloon method, we could safely remove the entrapped fragments of cath-lab devices from the coronary system in a very short time. Furthermore, the balloon technique has the advantage of simplicity and it also evades any additional cost to the patient. We suggest that balloon/guide ratio should be $>1$ and the appropriate inflation pressure for the retrieval balloon should be 10-14 atm, so that the balloon can hold the broken/dislodged fragments against the inner wall of the guiding catheter with enough pressure to enable us to pull the whole system en masse. We believe that the balloonassisted retrieval method is a simple and safe way to avoid complications of endothelial injury, myocardial infarction, emergency coronary artery bypass graft, and sudden cardiac death, as it does not require any manipulation in the heart or introduction of any other hardware inside the heart.

\section{Conclusions}

The balloon-assisted retrieval method is a simple, safe, and cost-effective method for device retrieval during cardiac procedures. We recommend the use of simple balloon method for the effective retrieval of broken/ dislodged balloons, guidewires, and stents during cardiac procedures in the cath-lab. However, it is strongly suggested that the choice of retrieval technique should be specific to each case and should be based on operator experience as well as availability of retrieval devices.

$$
\text { *** }
$$

Funding sources: No financial support was received for this study.

Authors' contribution: SP, RR, and CNM contributed in interventional procedure and final approval of the version to be published. NS and JM contributed in literature review, drafting of the manuscript, and final approval of the version to be published. All authors had full access to all data in the study and take responsibility for the integrity of the data and the accuracy of the data analysis.

Conflict of interest: The authors declare no conflict of interest.

Acknowledgements: The work was performed at Department of Cardiology, Sri Jayadeva Institute of Cardiovascular Sciences and Research, Bangalore, Karnataka, India.

\section{References}

1. Surhonne PS, Mahla H, Bhairappa S, Somanna S, Manjunath CN: Successful retrieval of fractured pressure wire tip (FFR) by hybrid technique. J Saudi Heart Assoc 27, 118-122 (2015)

2. Mehta V, Pandit BN, Trehan V: Retrieval of broken export catheter during primary angioplasty. Int J Angiol 22, 185-188 (2013)

3. Patil S, Ramalingam R, Kharge J, Nayak M, Manjunath $\mathrm{CN}$ : Successful retrieval of uncoiled coronary guidewire using simple balloon method. J Clin Diagn Res 9, OD01-OD03 (2015) 
Patil et al.

4. Patil S, Agarwal A, Ramalingam R, Kumar T, Agarwal N, Manjunath $\mathrm{CN}$ : Successful percutaneous removal of broken mitral valvuloplasty coiled tip guidewire. Cardiovasc Interv Ther 28, 398-402 (2013)

5. Kharge J, Sreekumar P, Swamy K, Bharatha A, Ramegowda RT, Nanjappa MC: Balloon-assisted retrieval of a broken stent-delivery system. Tex Heart Inst J 39, 644-646 (2012)

6. Patel T, Shah S, Pandya R, Sanghvi K, Fonseca K: Broken guidewire fragment: A simplified retrieval technique. Catheter Cardiovasc Interv $51,483-486$ (2000)

7. Singh D, Darbari A: Retrieval of trapped and broken guide wire with immediate rescue off-pump coronary bypass surgery. Interact Cardiovasc Thorac Surg 19, 529-531 (2014)
8. Chang WT, Chen JY, Li YH, Tsai LM, Lee CH: A two-case series of entrapment of a ruptured balloon in the coronary artery: Avoidable complications and nonsurgical management. J Formos Med Assoc $114,1135-1139(2015)$

9. Brilakis ES, Best PJ, Elesber AA, Barsness GW, Lennon RJ, Holmes DR Jr, Rihal CS, Garratt KN: Incidence, retrieval methods, and outcomes of stent loss during percutaneous coronary intervention: A large single-center experience. Catheter Cardiovasc Interv 66, 333-340 (2005)

10. Alomar ME, Michael TT, Patel VG, Altomare CG, Rangan BV, Cipher D, Banerjee S, Brilakis ES: Stent loss and retrieval during percutaneous coronary interventions: A systematic review and meta-analysis. J Invasive Cardiol 25, 637-641 (2013) 